\title{
Detector Solenoid Compensation in the PEP-II B-Factory*
}

\author{
Y. Nosochkov, Y. Cai, J. Irwin and M. Sullivan \\ Stanford Linear Accelerator Center, Stanford University, Stanford, CA 94309 USA \\ E. Forest \\ Lawrence Berkeley Laboratory, University of California, Berkeley, CA 94720 USA
}

\begin{abstract}
The PEP-II experimental detector includes a strong 1.5 $\mathrm{T}$ solenoid field in the interaction region (IR). With the fringe fields, the solenoid extends over a range of $6 \mathrm{~m}$. Additional complications are that 1 ) it is displaced longitudinally from the interaction point (IP) by about $40 \mathrm{~cm}$, 2) neither beam is parallel to the solenoid axis, and 3) the solenoid overlaps a dipole and a quadrupole on either side of the IP. In each half IR the correction system includes a set of skew quadrupoles, dipole correctors and normal quadrupoles to independently compensate the coupling, orbit perturbation, dispersion and focusing effect produced by the solenoid. The correction schemes for the Low Energy Ring (LER) and for the High Energy Ring (HER) are described, and the impact on the dynamic aperture is evaluated.
\end{abstract}

\section{INTRODUCTION}

The current design of the PEP-II experimental detector calls for a $1.5 \mathrm{~T}$ solenoid field. The solenoid length, including fringe fields, is about $6 \mathrm{~m}$, and the total integrated field is $5.7 \mathrm{Tm}$. The different beam energies, $3.1 \mathrm{GeV}$ for positrons and $9 \mathrm{GeV}$ for electrons, mean that displacing the solenoid center about $40 \mathrm{~cm}$ in the direction of the high energy beam (HEB) improves the detector acceptance of the particles produced in the beam collision. The solenoid overlaps a horizontal dipole B1 and the first final doublet (FD) quadrupole QD1 on each side of the IP. Therefore a superposition of magnetic fields exists in these magnets.

The beams are horizontally separated in $\mathrm{B} 1$ and in QD1 making the trajectories of the two beams neither identical nor parallel to the solenoid field. The resulting angle between the beams and the solenoid axis leads to a perturbation of the vertical orbit. The low energy beam (LEB), generally, experiences a larger orbit perturbation. With an antisymmetric beam trajectory about the IP, the effect on the LEB can be reduced by adjusting the horizontal tilt angle between the solenoid direction and the beam at the IP. This will bring the LEB closer to the solenoid axis and will provide partial cancellation of the orbit perturbation. The optimum tilt angle for the PEP-II solenoid is about 20 mrad which provides a compromise for the orbits of both beams. The top view of the solenoid with respect to the beam trajectories is shown in Figure 1.

*Work supported by the Department of Energy under Contract No. DE-ACO3-76SF00515 and DE-AC03-76SF00098.

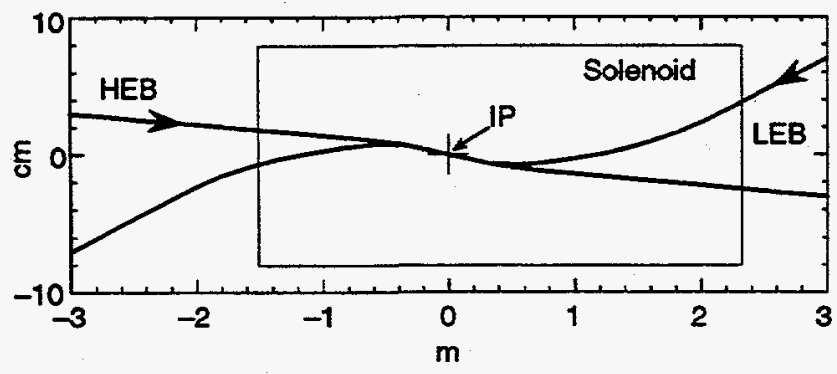

Figure 1. Top view of the beam orbits in the solenoid.

\section{SOLENOID FIELD MODEL}

In our calculations we used a simplified solenoidal field model which assumes a constant $1.5 \mathrm{~T}$ field over the effective length of $3.8 \mathrm{~m}$. Figure 2 shows the shape of the model (solid line) and of the realistic field (dash). The main difference is that the realistic field has an extended soft fringe field while the model solenoid has hard edges.

We used the MAD code [2] for numerical analysis. Currently, there is no provision in MAD for a quadrupole or a dipole superimposed with a solenoid, so we used an approximation to model the lattice. Within the solenoid, the B1 and QD1 magnets were replaced by the same length solenoid magnets sliced in $5 \mathrm{~cm}$ pieces, and a thin lens dipole or a quadrupole magnet was placed in the middle of each piece to produce the effect of B1 or QD1. The solenoid pieces in the model are aligned along the beam and will not generate orbit perturbations. To simulate the orbit effect, additional thin lens orbit kickers were uniformly distributed through out the solenoid. The kicker strength was calculated according to the beam orientation in the solenoid.

At the end of the solenoid the beams are not centered on the solenoid axis and, thus, receive a vertical orbit kick from the fringe field. In the model we simulated an offcentered fringe field by using a coordinate transformation in MAD.

The orbit effect was verified independently with the code MAGBENDS [3] which uses the superimposed mag-

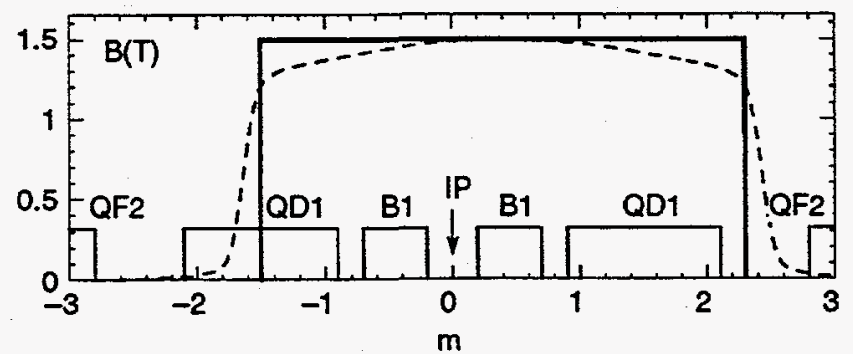

Figure 2. The model and the realistic solenoid field. 
netic field from all elements, and a realistic solenoid fringe field. There is reasonable agreement between the two models, and the difference can be accounted for by different fringe models.

\section{CORRECTION STRATEGY}

The following solenoid effects need to be corrected: 1) the coupling between $x$ and $y$ betatron oscillations, 2) vertical and horizontal orbit perturbations, 3) vertical dispersion distortion, and 4) solenoid focusing. Our goal was to cancel these effects at the IP and everywhere outside the IR. The asymmetry of the solenoid requires an independent local correction system on each side of the IP. Generally, for each half IR we need: 1) six skew quadrupoles to compensate the coupling and vertical dispersion, 2) two vertical and two horizontal orbit correctors, and 3) eight variable normal quadrupoles to match the Twiss functions and the horizontal dispersion.

Due to the simplecticity of a transfer matrix there are four independent coupling coefficients. In matrix formalism the betatron coupling is described by the off-diagonal $2 \times 2$ matrix in the $4 \times 4$ transfer matrix. Therefore, for local coupling correction we need to cancel the $(1,3),(1,4),(2,3)$ and $(2,4)$ terms in the transfer matrix between the IP and the end of the IR. Similarly, the vertical dispersion can be described by $(3,6)$ and $(4,6)$ terms in a $6 \times 6$ matrix.

For analysis of the optimum skew quadrupole positions we used the projection method developed in Reference [1]. In this approach, an original lattice with distributed coupling elements is replaced by an equivalent lattice with the same transfer matrix, but with all coupling elements placed at one point, usually the IP. The matrices of the coupling elements have to be modified after this transformation. For instance, an original matrix $Q$ of a thin skew quadrupole will be replaced by a new matrix $R=M^{-1} Q M$, where $M$ is an uncoupled matrix from the IP to the original position of the quadrupole. With all coupling elements placed at one point, the rest of the lattice is decoupled, and the coupling terms will add at the IP. It is, therefore, easy to analyze the effect from different coupling elements.

Consider one half of the IR starting from the IP. Suppose the Twiss functions at the original position of a thin skew quadrupole are $\beta, \alpha$ and $\mu$. The equivalent quadrupole at the IP will have a new transfer matrix with the following terms:

$$
\begin{aligned}
& R_{13}=q \sqrt{\frac{\beta_{x}{ }^{*}}{\beta_{y}{ }^{*}}} S_{x} C_{y}, \quad R_{14}=q \sqrt{\beta_{x}{ }^{*} \beta_{y}{ }^{*}} S_{x} S_{y}, \\
& R_{23}=-q \frac{C_{x} C_{y}}{\sqrt{\beta_{x}^{*} \beta_{y}^{*}}}, \quad R_{24}=-q \sqrt{\frac{\beta_{y}^{*} \beta_{x}^{*}}{\beta_{x}^{*}}} C_{x} S_{y}, \\
& R_{36}=f D_{x} \sqrt{\beta_{y} \beta_{y}^{*}} S_{y}, \quad R_{46}=-f D_{x} \sqrt{\frac{\beta_{y}}{\beta_{y}^{*}}} C_{y},
\end{aligned}
$$

where $f=\frac{L_{q}}{B_{p}} \frac{d B_{x}}{d x}$ is a quadrupole strength, $q=f \sqrt{\beta_{x} \beta_{y}}$, $\beta^{*}$ is a $\beta$-function at the IP, $C=\cos \mu, S=\sin \mu$ and $D_{x}$ is the horizontal dispersion at the original position of a skew quadrupole. The optimum positions for four coupling correctors will be at $\left(\mu_{x}, \mu_{y}\right)=(\pi / 2,0),(\pi / 2, \pi / 2),(0,0)$ and $(0, \pi / 2)(\bmod \pi)$, in which case each skew quadrupole generates only one non-zero coupling term at the IP, thus providing an orthogonal correction. The other two skew quadrupoles will provide an orthogonal vertical dispersion correction if placed at $\mu_{y}=0$ and $\pi / 2(\bmod \pi)$ where $D_{x}$ is large.

In a similar way, the solenoid can be moved to the IP. The projected solenoid transfer matrix, which can be calculated numerically using $\mathrm{MAD}$, is equal to $S=M_{o f f}{ }^{-1} M_{o n}$, where $M$ is a matrix from the IP to the end of a solenoid, for the solenoid turned off and on, respectively. For a nonoverlapped solenoid only two major coupling terms would exist, $(1,3)$ and $(2,4)$, which is just the rotation angle of the betatron planes. The interaction between the solenoid and the quadrupole gives rise to the other two coupling terms. Figure 3 shows how these terms depend on the overlap with the B1 and QD1 magnets. The results correspond to the longer side of the solenoid in the LER, where the effect on the beam is the largest. In Figure 3 the solenoid starts at the IP and its length $L$ is varied from $0.5 \mathrm{~m}$ to the nominal value of $2.3 \mathrm{~m}$, while the integrated field stays constant at $3.45 \mathrm{Tm}$. For a non-overlapping solenoid (dashed line) the coupling terms are almost constant with $L$. If the $\mathrm{B} 1$ and QD1 magnets are present, then the terms start changing after the solenoid expands into QD1 located at $0.9 \mathrm{~m}$ from the IP (solid line).

Figure 4 shows the skew quadrupole terms projected to the IP as a function of position in the IR. The terms vary according to formulas (1), and the skew quadrupole strength was fixed at $10 \%$ of the main arc quadrupole strength. The corresponding solenoid term at the IP is shown by a dashed line. Note, that the hardest solenoid term to correct in this case is the $(1,4)$ term. It is generated from the overlapped QD1 and grows quadratically with the overlapped length. This term is much smaller for the shorter side of the solenoid since QD1 is not fully overlapped on this side. In a real lattice it is usually not possible to find the ideal phase positions for the quadrupoles. Therefore, all six quadrupoles may generate all six terms, and a combined set of equations has to be solved.

To locally compensate the orbit at the IP, the general strategy is to use two dipole correctors for each half IR and

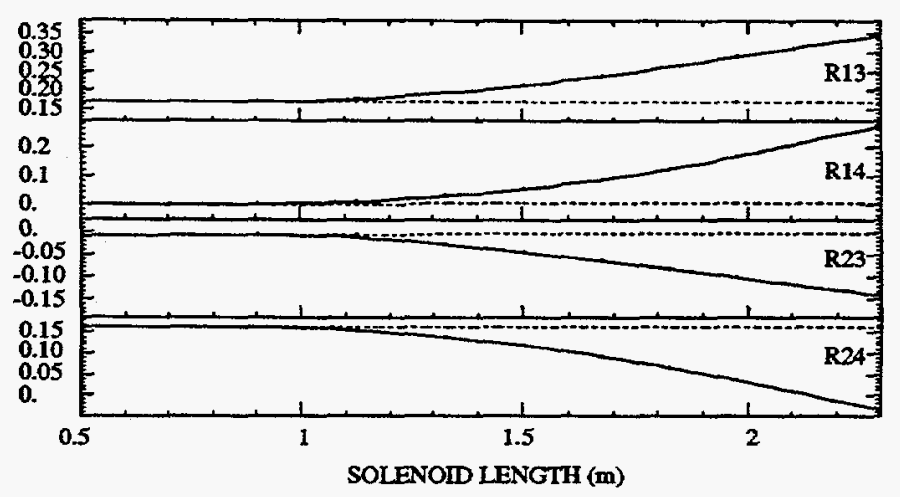

Figure 3. Projected solenoid coupling terms at the IP. 


\section{DISCLAIMER}

This report was prepared as an account of work sponsored by an agency of the United States Government. Neither the United States Government nor any agency thereof, nor any of their employees, makes any warranty, express or implied, or assumes any legal liability or responsibility for the accuracy, completeness, or usefulness of any information, apparatus, product, or process disclosed, or represents that its use would not infringe privately owned rights. Reference herein to any specific commercial product, process, or service by trade name, trademark, manufacturer, or otherwise does not necessarily constitute or imply its endorsement, recommendation, or favoring by the United States Government or any agency thereof. The views and opinions of authors expressed herein do not necessarily state or reflect those of the United States Government or any agency thereof. 


\section{DISCLAIMER}

Portions of this document may be illegible in electronic image products. Images are produced from the best available original document. 


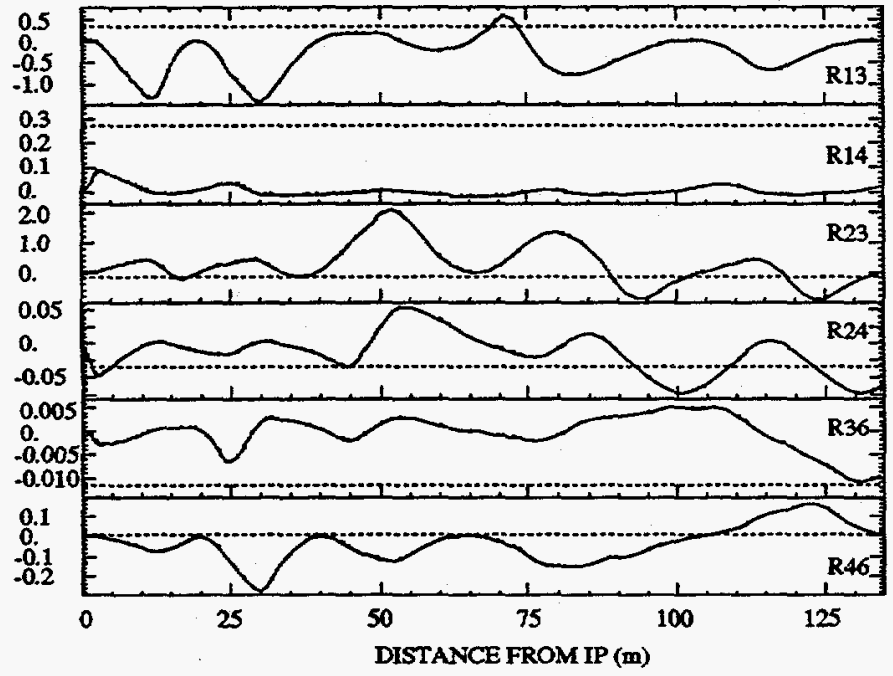

Figure 4. Projected skew quadrupole terms at the IP.

for each betatron plane, and to locate them at orthogonal phase positions of 0 and $\pi / 2(\bmod \pi)$ from the IP.

Finally, the focusing perturbation has to be corrected with a minimum of eight variable normal quadrupoles in each half IR. The solenoid focuses in both planes. For the LEB it generates a quadrupole component that is about $1 \%$ of the QD1 strength. The skew quadrupoles affect the optics as well. More normal quadrupoles can be varied if additional constraints are imposed. For instance, in order to minimize perturbation of the chromaticity correction, we kept the $\beta$-functions and phases unchanged at the local sextupole positions with the solenoid on. In all, in each half IR we adjusted the two FD quadrupoles by $1-2 \%$ in strength, 11 normal quadrupoles in the LER, and 9 quadrupoles in the HER.

\section{RESULTS}

We tried a variety of possible skew quadrupole positions in order to minimize their maximum strength. Skew quadrupole positions next to the local sextupoles are effective because of the large $\beta$ function and because the $-I$ transformation between the two skew quadrupoles makes it possible to independently control the coupling and the vertical dispersion. On the longer side of the solenoid the important skew quadrupole position is near the final doublet, where the $\beta$ values are large and the phase provides the best correction of the $(1,4)$ term. Because of the large orbit perturbation, the first vertical orbit corrector was placed near the FD. We did not place any elements for the solenoid correction between the paired local sextupoles in order to preserve the $-I$ transformation. The positions of the skew quadrupoles and of the orbit correctors on two sides of the IR are symmetric about the IP.

All correction strengths were calculated numerically using MAD. Table 1 shows the skew quadrupole strengths for the two rings that independently compensate the long and the short side of the solenoid. The strongest skew

\begin{tabular}{|c|c|c|c|c|}
\hline \multirow{2}{*}{$\begin{array}{c}\text { Corrector } \\
\text { name }\end{array}$} & \multicolumn{2}{|c|}{ LER } & \multicolumn{2}{c|}{ HER } \\
\cline { 2 - 5 } & Long & Short & Long & Short \\
\hline \hline SQ1 & -0.07210 & 0.01479 & -0.00389 & 0.00076 \\
SQ2 & 0.02405 & -0.00502 & 0.00369 & -0.00110 \\
SQ3 & -0.00434 & -0.00052 & -0.00039 & -0.00003 \\
SQ4 & 0.00271 & -0.00110 & 0.00054 & 0.00012 \\
SQ5 & 0.02638 & -0.02020 & 0.00861 & -0.00455 \\
SQ6 & 0.00866 & -0.01176 & 0.00202 & -0.00360 \\
\hline
\end{tabular}

Table 1: Skew quadrupole strength $\frac{L_{q}}{B \rho} \frac{d B_{x}}{d x}\left(m^{-1}\right)$.

quadrupole occurs in the LER near the final doublet, on the longer side of the solenoid, to correct the $(1,4)$ term.

Orbit perturbations caused by the solenoid in the LER are shown in Figure 5. If a soft fringe field is included instead of the hard solenoid edges, then the maximum vertical orbit would be slightly larger than $6 \mathrm{~mm}$.

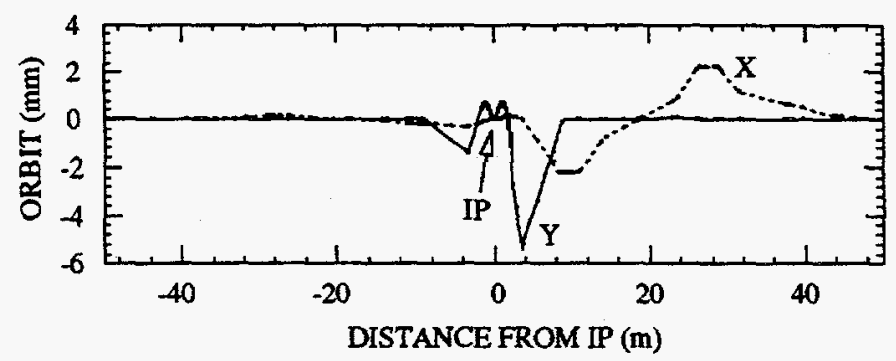

Figure 5. Orbit due to solenoid in the LER.

Finally, the effect of the solenoid on dynamic aperture was evaluated. Typical reduction of the aperture due to the solenoid is about $2-4 \sigma$, and the final aperture including machine errors is close to $10 \sigma$. An example of the LER short term dynamic aperture with the solenoid is shown in Figure 6.

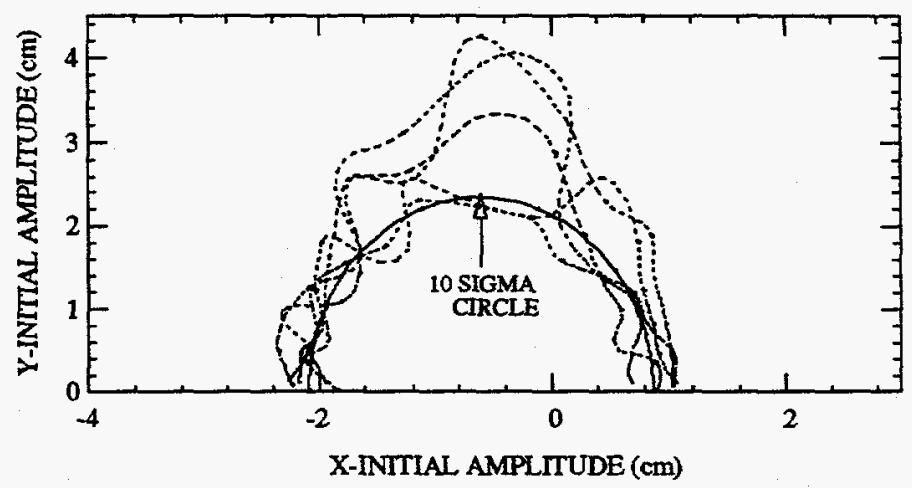

Figure 6. LER dynamic aperture for 5 seeds (dash).

\section{References}

[1] S. Peggs, "The Projection Approach to Solenoid Compensation", CERN/SPS/82-2 (DI/MST) (1982).

[2] H. Grote, F.C. Iselin, "The MAD Program, Version 8.1", CERN/SL/90-13 (AP) (1990).

[3] M. Sullivan, unpublished. 